\title{
Reflexiones sobre la teoría del turismo y su enfoque disciplinar
}

\author{
Reflections on the theory of tourism and its disciplinary approach
}

\author{
Abraham Briones-Juarez ${ }^{a}$, Erika Cruz Coria ${ }^{b}$
}

\begin{abstract}
:
This essay reflects on the social and administrative approach of tourism to locate their differences and expose the need to generate a series of basic principles that can support the advance of tourism theory. The progress in the study of tourism should allow us, both the identification of new techniques and the advance in their theoretical foundations. Therefore, the study of tourism should focus on developing its own foundations and expanding its ontological and epistemological presentation.
\end{abstract}

Keywords:

Tourism, Discipline, Epistemology

\section{Resumen:}

Este ensayo reflexiona sobre el enfoque social y administrativo del turismo para ubicar sus diferencias y exponer la necesidad de generar una serie de principios básicos que puedan apoyar en el avance de la teoría del turismo. El progreso en el estudio del turismo debe permitir, tanto la identificación de nuevas técnicas como el avance en sus fundamentos teóricos. Por lo tanto, el estudio del turismo debe centrarse en desarrollar sus propios fundamentos y ampliar su presentación ontológica y epistemológica.

Palabras Clave:

Turismo, Disciplina, Epistemología

\section{Introducción}

Para introducir, los aspectos disciplinares y multidisciplinares del turismo se discute la necesidad de emprender dicha empresa. Por un lado, el turismo se nutren de las necesidades de conocimiento del campo profesional y disciplinar, las cuales llegan a concretarse en una orientación curricular [1], esta necesidad ha propiciado diferencias en el estudio de lo turístico. Las disciplinas asociadas con el turismo se presentan como esquemas de clasificación en las cuales se utilizan conocimientos probados para ser replicados en entornos partículas. Sin embargo, el estudio del turismo resulta de cierta complejidad al integrar entornos, recursos, medios, elementos y contextos que advierten la identificación de medio, métodos y metodologías de vanguardia para tratar de comprenderlo y de ser el caso proponer nuevas formas para su abordaje científico.

\section{DESARROLLO.}

Las disciplinas que integran el estudio del turismo han sido clasificadas por algunos autores, por ejemplo, Guevara, Molina, y Tresserras, [2] hacen una separación de las ciencias sociales con especial énfasis en el área de las humanidades, y las ciencias naturales que se utilizan para diseñar y controlar la actividad turística. Por su parte Mota [3], desarrolla una una subclasificación de las disciplinas del turismo en a) disciplinas con aplicación al conocimiento del turismo, b) disciplinas con capacidad limitada para los abordaje del turismo c) disciplinas que no pueden ser adaptadas a lo turístico. De la misma forma, Butowski [4], propone a partir de las áreas de interés del turismo una división de disciplinas en torno a una temática compuesta, y señala a los siguientes aspectos para integran diversas disciplinas de estudio: a) la organización del fenómeno, b) los flujos, c) los desplazamientos (destinos y de tránsito), d) las formas de desarrollar la actividad d) y efectos o consecuencias.

Así, el turismo se ubica en las ciencias sociales y se apoya de diversos conocimientos, que se ubican de acuerdo a las actuales exigencias en el campo. El turismo mantiene

${ }^{a}$ Autor de Correspondencia, Universidad Autónoma del Estado de Hidalgo, Instituto de Ciencias Económico Administrativas, ORCID: 00000002-3124-8741,Email: abraham_briones6894@uaeh.edu.mx

${ }^{\text {b } U n i v e r s i d a d ~ A u t o ́ n o m a ~ d e l ~ E s t a d o ~ d e ~ H i d a l g o, ~ I n s t i t u t o ~ d e ~ C i e n c i a s ~ E c o n o ́ m i c o ~ A d m i n i s t r a t i v a s, ~ E m a i l: ~ e c o r i a 84 @ h o t m a i l . c o m ~}$ 
un carácter dinámico que han generado cambios, a los cuales deben de responder los profesionales [5], y que incluyen habilidades compatibles de acuerdo a los nuevos retos que afrontan [6]. De tal forma, se promueve el pensamiento crítico, dada su naturaleza, lo que presenta nuevos desafíos a medida de que el turismo avanza hacia nuevos frentes científicos y ocupa un lugar frente a campos ya establecidos [7].

Sin embargo, el turismo no se puede atribuir a una sola disciplina teórica. El avance de su conocimiento se retoma por teorías parciales que impulsan el desarrollo investigativo y práctico [8]. Así, el abordaje del turismo intuye el poner de manifiesto las disciplinas compatibles, ya que cada una de sus áreas de conocimiento se vierten en una forma particular para constituirlo, lo que [9] señala como la definición parcial.

El turismo es en esencia un fenómeno social que se centra en los desplazamientos de personas, y se fundamenta en la búsqueda de la naturaleza y los efectos que desprende, los cuales pueden ser sociales y/o productivos como lo apunta [10], la complejidad del estudio del turismo resalta en la importancia de su conocimiento con la aportación multidisciplinar del resto de disciplinas que interactúan en el sistema turístico. De aquí una derivación importante, la separación en un enfoque dual que pone de manifiesto las principales apariencias del turismo.

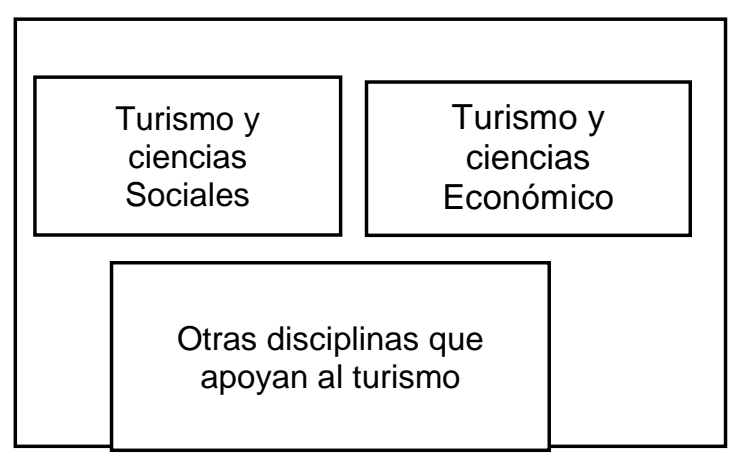

Figura 1 disciplinas que apoyan la actividad turísticas.

La parte social del turismo se gesta a lo largo de un camino de teorización que comenzó a inicio del siglo XIX, con la sociología en Europa interesada en las motivaciones, los roles, las relaciones y el impacto del turismo en las comunidades [11], El mismo camino corrió la turismo antropológico [12] con apoyo a la conformación de materia de visita e integración de atractivos. En esta corriente, el turismo se comprende en su enfoque humanístico de contacto entre culturas [13] y con una dialéctica etno-cultural [14].

En la sociología el turismo y sus constructos como el espacio, la relación social y el entorno se han teorizado predominantemente de acuerdo al constructivismo social [15]. Así, el ocio se constituye por las dimensiones existencial y social no determinadas [16]. A partir de ello, los autores trazan el desarrollo de la teoría sociológica y la aparición de una variedad de enfoques [17], como la sociología cualitativa de tradiciones [18] y el pluralismo teórico [19]. En la construcción social el turismo es ambas un fenómeno cultural [20] con plataforma de disposición en su práctica y un fenómeno social comprendido a partir del ejercicio de los roles fundamentales [21]. Por su parte, en la antropología del turismo [22] propone las manifestaciones superestructurales que principian de una antropología multicultural para entenderlo.

La vertiente social del turismo de disciplinas socioantropológico extiende el camino de la teorización, sin embargo, se limitan a la abstracción de las ideas. Por tanto, no garantiza su explicación empírica.

Por otro lado, se ubican las disciplinas que apoyan las soluciones productivas a través de la gestión de los recursos, y que proceden de la administración científica, la cual modifico los servicios turísticos a principios del siglo $X X$ [23], en este contexto el turismo deviene del modelo fordista [24] cuya manifestación permite suponer un arreglo de recursos para cumplir objetivos. Esta noción dio paso al turismo postmoderno en el que la producción se hace más complicada con una serie de actividades relacionadas [25]. Lo que Sergio [26], llama el nuevo paradigma del pos-turismo que incluye cambios en la sociedad y la tecnología. Estos elementos (tecnología y masificación) generan nuevos productos que se reproducen en una relación de capitales [27].

La vertiente productiva a diferencia de la social, acarrea la ventaja de que no se considera necesario dar mayor extensión teórica para desarrollar aplicaciones, sino que basta con elegir postulados ya probados en otras áreas de conocimiento, tal es el caso de la administración y la economía.

Por ejemplo en su estudio [28], analizan la estructura de la industria hotelera, utilizando un análisis de las cinco fuerzas de Porter, para desprender el grado de competitividad del sector. Sin embargo, este estudio no parte de las relaciones de lo turístico, sino de la teoría de la administración y supone además que las empresas hoteleras se componen de recursos enlazados que vierten un grado de competitividad. Así, el turismo deja de tener una esencia múltiple y se convierte en una temática de abordaje disciplinar, en la cual se identifica una disciplina y sus modelos, métodos, teorías, y/o técnicas convenientes para explicar el fenómeno. Es fácil imaginar que con todo este conglomerado de conocimientos, los 
estudios refieren a la solución productiva, ya que se centran en la búsqueda de resultados, más que en enfocarse sobre las posibles dificultades para hacer esta materialización. La misma suerte corren los ejemplos de la tabla 1.

\begin{tabular}{|c|c|c|c|c|}
\hline $\begin{array}{l}\text { Estudios de } \\
\text { lo turístico }\end{array}$ & Disciplina & \begin{tabular}{|l} 
Método/ \\
teoría
\end{tabular} & Técnica & Autor (es) \\
\hline $\begin{array}{l}\text { Intercambio } \\
\text { de clientes } \\
\text { con marcas } \\
\text { de medios } \\
\text { sociales de } \\
\text { turismo }\end{array}$ & Mercadotecnia & $\begin{array}{l}\text { Modelo } \\
\text { Estructural }\end{array}$ & $\begin{array}{l}\text { Escala de } \\
\text { compromiso }\end{array}$ & $\begin{array}{l}\text { (Harrigan, } \\
\text { Evers, } \\
\text { Miles, \& } \\
\text { Daly, } \\
2017 \text { ) } \\
{[29]} \\
\end{array}$ \\
\hline $\begin{array}{l}\text { Las } \\
\text { decisiones } \\
\text { de precios } \\
\text { estratégicas } \\
\text { y tácticas } \\
\text { en gestión de } \\
\text { ingresos } \\
\text { hoteleros }\end{array}$ & Economía & $\begin{array}{l}\text { Variables, } \\
\text { clasificadas } \\
\text { según su } \\
\text { naturaleza } \\
\text { tangible o } \\
\text { contextual. }\end{array}$ & $\begin{array}{l}\text { Técnicas } \\
\text { dinámicas } \\
\text { de fijación } \\
\text { de precios }\end{array}$ & $\begin{array}{l}\text { (Abrate \& } \\
\text { Viglia, } \\
2016) \\
{[30]}\end{array}$ \\
\hline $\begin{array}{l}\text { Recursos } \\
\text { humanos }\end{array}$ & Administración & $\begin{array}{l}\text { Análisis de } \\
\text { tabulación } \\
\text { cruzada }\end{array}$ & $\begin{array}{l}\text { Grupos } \\
\text { focales y } \\
\text { entrevistas } \\
\text { personales }\end{array}$ & $\begin{array}{l}\text { (Pizam, } \\
\text { 1999) } \\
{[31]}\end{array}$ \\
\hline
\end{tabular}

\section{Tabla 1. Estudios disciplinares en Administración en las organizaciones turísticas.}

Otros apartados de uso teórico específico, se aplican por añadidura al campo, por ejemplo la informática y la contabilidad que advierten en la colectividad desarrollos y aplicaciones de especialistas desde su propia área de estudio para generar, supervisar o aplicar sus propios conocimientos o propuestas, caso específico lo constituyen los trabajos de Yuan, Xu, Qian, y Li [32], y Chunchang \& Nan [33], con modelos de información.

En consecuencia la compatibilidad que tiene el turismo con las distintas áreas del conocimiento muestra su versatilidad para mantener las propiedades y herramientas asociadas a los campos más desarrollados [34]. No obstante, sigue habiendo una notable ausencia de discusión sobre la investigación conceptual [35].

El gran faltante que se tiene en la teoría se encuentra en la conformación propia de los teóricos del turismo; es decir, en la presentación de ideas particulares y novedosas que puedan cumplir con las distintas fases del conocimiento. Lo cual incluye: a) la explicación de lo que trata el constructo del turismo, b) la teorización explicativa de donde se deprende los fundamentos, c) su pertinencia de utilización investigativa, d) su comprobación lógica y e) la oportunidad de decretar nuevos conocimientos que nutran la teoría. Es decir el compuesto que Mota [3], Ilama los medios multidisciplinar, Interdisciplinar y transdisciplinar. La primera para enfocar el problema, la segunda para agrupar conceptos y teorías y el tercero para lidiar con la complejidad del fenómeno.

La integración disciplinaria del turismo de acuerdo con Guerrero y Gallucci [36], es un camino que permite la comprensión del objeto de estudio, y argumenta: para ello, se debe generar el enfoque propio que permita un marco conceptual y la terminología propia.

Partiendo de la base anterior, se puede concretar el conocimiento y dar pasos de firmeza que apunten no solamente a una forma propia de explicación, sino a su conjunción continua con aportaciones separadas o no que pueden más fácilmente probarse en el plano empírico.

En otros campos del conocimiento los autores han tomado el reto de teorizar y establecer la pauta para trascender en la explicación de los fenómenos, ejemplo de ello son los trabajos de Samuelson [37], en economía, Ackoff [38], en la administración y Durkheim [39], en sociología etc. Los planteamientos primarios determinan la sigilosa cautela con la que se vierten los contenidos que toda vez que cumplen con el propósito de ser refutados, se solidifican. Sin embargo, en el caso del turismo, los autores han seguido distintos caminos, hay desde los teóricos puros como Cohen, [40] que estudio la tipología del turismo, Leiper [41], pionero en el modelado pronunciado en una visión teórica holística a través de elementos geográficos y la relación con la industria. Con la Teoría de la aculturación [42] con un enfoque semiótico que explica la realidad social y el contacto de clases. Graburn [43], que propone desde la antropología el turismo simbólico lleno de expresiones culturales. Pearce [44], que estudio las motivaciones y los contactos interculturales.

Por otro lado algunos autores han tratado de indagar sobre la dinámica del turismo para ubicar estructuras de comportamiento como Pearce, [45], que a través de la estadística observó el grado de aceptación de los turistas en zonas urbanas y rurales o como Jafari [46], que retoma el turismo como industria para ubicar sus elementos importantes a través de la ampliación de su ámbito.

Sin embargo, no se ha tenido una aceptación general salvo de la teoría clásica del turismo que siempre que es abordada se recicla como receta tasita y que no ha sufrido gran cambio desde que fue planteada, es decir con los autores clásicos que la concretaron. Así, la teoría nace en un momento en la que no se cree pertinente esclarecer su trascendencia a la praxis. Estos esfuerzos sin embargo son significativos para comprender la esencia básica del turismo, más no para interpretar la realidad a partir de los mismos.

De igual manera, la moda en la tendencia explicativa de distintas realidades ha influido en la temática del turismo, se habla de sustentabilidad, modelo alternativo y armonía de los actores. Así, se han utilizado los enfoques derivados que siguen perteneciendo en esencia a teorías 
distintivas, útiles pero distintas a la esencia que emana del turismo.

La concreción conceptual debe desprenderse de la filosofía de las ciencias ya que refiere a un contexto de aplicación que decanta en una taxonomía y que a su vez deriva ontológicamente en una epistemología. El conocimiento así; es decir la síntesis deviene al utilizar los componentes anteriores a partir de una formulación propia.

De esta forma, de ser el caso de que el conocimiento del turismo no requiere de la presencia y definición de su esencia. La ciencia secular trasciende y los autores se apartan de definir postulados propios.

Por otro lado, si la esencia es parte de los estudios, a los investigadores les corresponde hacer la presentación sistemática del caso.

No obstante, los fundamentos disciplinares se pueden incluso aplicar de forma arbitraria y cambiar por completo la problemática que representan, es decir, constituyendo solo una visión argumentativa de su contemplación. En este sentido el avance teórico derivado de las disciplinas y la praxis del turismo se moderan con base en la disciplina que lo describe. Por otro lado, la conformación del turismo como una disciplina propia ha llevado a la misma determinación, que el turismo constituye un entramado no definido y que de cualquier forma conviene comprenderlo y de la misma manera busca mecanismos que lo definan, con la finalidad de poder utilizar la teoría en la comprensión conceptual.

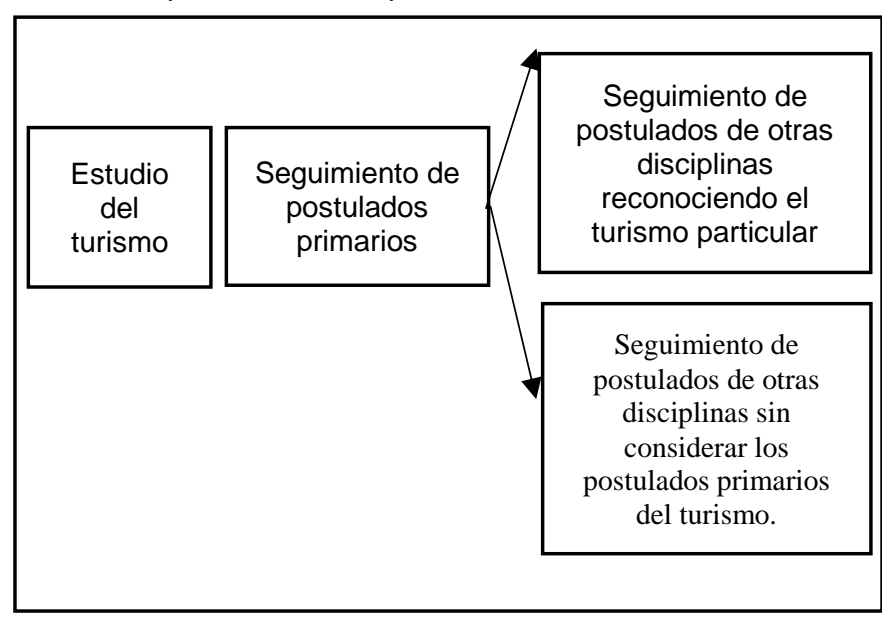

Figura 2 Postulados de lo turístico.

Así, existe una separación entre los propios fundamentos del turismo y su aplicación disciplinar; esta brecha, se va haciendo cada vez más notable, debido a que en los últimas décadas se ha detenido el avance teórico del estudios del turismo ya sea a través de la réplica de los elementos ya propuestos, del surgimiento de nuevos fundamentos que no se han valorizado para ser plenamente aplicados $o$ de la presentación de aportaciones desagregadas con poco impacto 0 aceptación de la academia.

\section{CONCLUSIONES}

El avance del conocimiento del turismo se ha comprendido a partir de dos condiciones, una utilización de disciplinas para tratar de explicarlo y el desarrollo de fundamentos para avanzar en sus definiciones teóricas. El primero, (las disciplinas que apoyan al turismo), no parten de su naturaleza fenomenológica que la caracteriza, ya que al ser utilizadas para explicar en parte lo que ocurre en la realidad. De esta forma, Las ciencias particulares y sus fundamentos de mayor avance y trascendencia han substituido la utilización de una teoría turística de mayor envergadura. Por otro lado la teoría social y antropológica vierte fundamentos conceptuales que en ocasiones están desagregados de lo que ocurre en la práctica del fenómeno. Por tanto, los estudiosos del turismo deben ubicar estas carencias y trabajar para superarlas al tiempo que se consolida una teoría turística más sofisticada.

\section{Referencias}

[1] Niding, M. (2004). Currícula de la licenciatura en Turismo y contextos sociales. Aportes y Transferencias, 1(8), 39-54.

[2] Guevara, R., Molina, S., \& Tresserras, J. (2006). HACIA UN ESTADO DE LA CUESTIÓN EN INVESTIGACIÓN TURÍSTICA. En Estudios Multidisciplinarios en Turismo (Vol. 1, pp. 17-64). México: Secretaría de Turismo. Recuperado a partir de http://bibliotecas.umar.mx/publicaciones/Estudios versión\%20electrón ica.pdf\#page $=18$

[3] Mota, K. (2004). La Integración Multidisciplinar en la Educación del Turismólogo. Estudios y Perspectivas en Turismo, 13, 5-23.

[4] Butowski, L. (2011). TOURISM - AN ACADEMIC DISCIPLINE (DISCURSIVE ARTICLE). Tourism, 21(1-2), 17-24. https://doi.org/10.2478/v10106-011-0002-8

[5] Trigo, L. G. G. (1998). A sociedade pós-industrial e o profissional em turismo. Papirus Editora.

[6] Stone, G. A., Duffy, L. N., Pinckney, H. P., \& Templeton-Bradley, R. (2017). Teaching for critical thinking: preparing hospitality and tourism students for careers in the twenty-first century. Journal of Teaching in $\begin{array}{llll}\text { Travel \& } & \text { Tourism, } & \text { 17(2), } & \text { 67-84. }\end{array}$ https://doi.org/10.1080/15313220.2017.1279036

[7] Jafary, J. (1994). La cientificación del turismo. Estudios y Perspectivas en Turismo, 3(1), 7-37.

[8] Liszewski, S. (2010). Tourism Studies: Situated within Multiple Disciplines or a single Independent Discipline? (discursive article). Tourism, Tourism Volume 20, Issue 2 (2010). Recuperado a partir de http://cejsh.icm.edu.pl/cejsh/element/bwmeta1.element.hdl $11089 \quad 114$ 39

[9] Onghena, Y. (2013). Introducción: ¿por qué una aproximación pluridisciplinar al turismo? Revista CIDOB d'Afers Internacionals, (113), 7-13.

[10] Fernández, J. I. P. (2006). ¿ESTÁ LA INVESTIGACIÓN EN TURISMO SUFICIENTEMENTE RECONOCIDA Y VALORADA EN ESPAÑA? REVISTA DE ANÁLISIS TURÍSTICO, 0(2). https://doi.org/10.1234/RAT2011n11 
[11] Apostolopoulos, Y., Leivadi, S., \& Yiannakis, A. (1996). The Sociology of Tourism: Theoretical and Empirical Investigations. Psychology Press.

[12] Jackowski, A., Sołjan, I., Bilska, E., \& Liro, J. (2016). Geographical tourism research and education at the Jagiellonian University School of Tourism in Poland (1936-1939). History of Geo- and Space Sciences, 7, 91-101. https://doi.org/10.5194/hgss-7-91-2016

[13] Castaño, J. M. (2005). Psicología social de los viajes y del turismo. Thomson.

[14] Ng, S. I., Lee, J. A., \& Soutar, G. N. (2007). Tourists' intention to visit a country: The impact of cultural distance. Tourism Management, 28(6), 1497-1506. https://doi.org/10.1016/j.tourman.2006.11.005

[15] Gren, M., \& Huijbens, E. H. (2012). Tourism theory and the earth. Annals of Tourism Research, 39(1), 155-170. https://doi.org/10.1016/j.annals.2011.05.009

[16] McCabe, S. (2014). Towards a better theory of tourism experience? A response to Gnoth and Matteucci. International Journal of Culture, Tourism and Hospitality Research, 8(2). https://doi.org/10.1108/IJCTHR-04-2014-0026

[17] Dann, G., \& Cohen, E. (1991). Sociology and tourism. Annals of Tourism Research, 18(1), 155-169. https://doi.org/10.1016/01607383(91)90045-D

[18] Cohen, E. (1988). Traditions in the qualitative sociology of tourism. Annals of Tourism Research, 15(1), 29-46. https://doi.org/10.1016/0160-7383(88)90069-2

[19] Cohen, E. (1979). Rethinking the sociology of tourism. Annals of Tourism Research, 6(1), 18-35. https://doi.org/10.1016/0160$\underline{\text { 7383(79)90092-6 }}$

[20] Talos, A.-M., \& Alexandru, B. P. (2011). Sociology of tourism. Cinq Continents, 1(2), 184-193.

[21] González, D. A. (2009). El turismo desde un enfoque de sociología constructivista. Teoría y praxis, 107-122.

[22] Nash, D., \& Smith, V. L. (1991). Anthropology and tourism. Annals of Tourism Research, 18(1), 12-25. https://doi.org/10.1016/01607383(91)90036-B

[23] Mentzer, M. S. (2010). Scientific Management and the American Hotel. Management \& Organizational History, 5(3-4), 428-446. https://doi.org/10.1177/1744935910361557

[24] Donaire, J. A. (1998). La reconstrucción de los espacios turísticos la geografía del turismo después del fordismo. Publicat a Sociedade e Territorio, 28, 2-34

[25] Nuryanti, W. (1996). Heritage and postmodern tourism. Annals of Tourism Research, 23(2), 249-260. https://doi.org/10.1016/01607383(95)00062-3

[26] Sergio, M. (2006). El Posturismo. Editorial Trillas Sa De Cv.

[27] Gastal, S. (2006). Tiempos postmodernos. Posibilidades para el turismo. Estudios y Perspectivas en Turismo, 15(3), 270-282.

[28] Carré, E. L., Saldaña, R. M. C., Salgado, P. M., \& León, A. T. (2012). Análisis De La Estructura De La Industria Hotelera. Caso: Hoteles De Negocios En La Ciudad De Toluca. Gestión Turística, (18), 147-180.

[29] Harrigan, P., Evers, U., Miles, M., \& Daly, T. (2017). Customer engagement with tourism social media brands. Tourism Management, 59, 597-609. https://doi.org/10.1016/j.tourman.2016.09.015

[30] Abrate, G., \& Viglia, G. (2016). Strategic and tactical price decisions in hotel revenue management. Tourism Management, 55, 123-132. https://doi.org/10.1016/j.tourman.2016.02.006

[31] Pizam, A. (1999). The state of travel and tourism human resources in Latin America. Tourism Management, 20(5), 575-586. https://doi.org/10.1016/S0261-5177(99)00026-6

[32] Yuan, H., Xu, H., Qian, Y., \& Li, Y. (2016). Make your travel smarter: Summarizing urban tourism information from massive blog data.
International Journal of Information Management, 36(6), 1306-1319. https://doi.org/10.1016/j.ijinfomgt.2016.02.009

[33] Chunchang, F., \& Nan, Z. (2012). The Design and Implement of Tourism Information System Based on GIS. Physics Procedia, 24, 528533. https://doi.org/10.1016/j.phpro.2012.02.077

[34] Jafari, J. (2005). El turismo como disciplina científica. Política y Sociedad, 42(1), 39-56.

[35] Xin, S., Tribe, J., \& Chambers, D. (2013). Conceptual research in tourism. Annals of Tourism Research, 41, 66-88. https://doi.org/10.1016/j.annals.2012.12.003

[36] Guerrero, A. L. del V., \& Gallucci, S. S. (2014). Aporte teórico conceptual al Turismo como disciplina académica a partir de la patrimonialización como proceso de valorización turística de los territorios. PASOS Revista de Turismo y Patrimonio Cultural, 13(1). Recuperado a partir de http://ojsull.webs.ull.es/ojs/index.php/Revista/article/view/320

[37] Samuelson, P. A. (2014). Paul Samuelson on the History of Economic Analysis: Selected Essays. Cambridge University Press.

[38] Ackoff, R. L. (1989). Cápsulas de Ackoff: administración en pequeñas dosis. Limusa.

[39] Durkheim, É. (1987). La división del trabajo social. Ediciones AKAL.

[40] Cohen, E. (1972). TOWARD A SOCIOLOGY OF INTERNATIONAL TOURISM. Social Research, 39(1), 164-182. https://doi.org/10.2307/40970087

[41] Leiper, N. (1979). The framework of tourism. Annals of Tourism Research, 6(4), 390-407. https://doi.org/10.1016/0160-7383(79)900033

[42] MacCannell, D. (1976). The Tourist: A New Theory of the Leisure Class. University of California Press.

[43] Graburn, N. H. H. (1989). 1. Tourism: The Sacred Journey. En Hosts and GuestsThe Anthropology of Tourism (Second Edition). Berlin, Boston: University of Pennsylvania Press. https://doi.org/10.9783/9780812208016.19

[44] Pearce, P. L. (1982). 1 - Tourists, Tourism and Tourist Psychology. En The Social Psychology of Tourist Behaviour (Vol. 3, pp. 1-25). Pergamon. https://doi.org/10.1016/B978-0-08-025794-5.50006-6

[45] Pearce, J. A. (1980). Host community acceptance of foreign tourists. Annals of Tourism Research, 7(2), 224-233. https://doi.org/10.1016/0160-7383(80)90005-5

[46] Jafari, J. (1974). The components and nature of tourism. Annals of Tourism Research, 1(3), 73-89. https://doi.org/10.1016/01607383(74)90034-6 